\title{
ON NON-TRIVIAL SPECTRA OF TRIVIAL GAUGE THEORIES
}

\author{
Piotr Korcyl ${ }^{\mathrm{a}, \mathrm{b}}$, Mateusz Koreń ${ }^{\mathrm{b}}$, JaceK WosieK ${ }^{\mathrm{b}}$ \\ ${ }^{a}$ NIC, DESY Zeuthen, Platanenallee 6, 15738 Zeuthen, Germany \\ ${ }^{\mathrm{b}}$ The Marian Smoluchowski Institute of Physics, Jagiellonian University \\ Reymonta 4, 30-059 Kraków, Poland
}

(Received March 4, 2013)

In this paper, we point out that the analytic solution of the two dimensional $U(1)$ gauge theory, on a finite lattice, reveals in the continuum limit the renowned Manton's spectrum of topological electric fluxes together with their effective Hamiltonian and wave functions. We extend this result for the system with strings and external charges providing also a novel interpretation of the $\Theta$ parameter. Some further generalizations are also outlined.

DOI:10.5506/APhysPolB.44.713

PACS numbers: 03.65.--w, 11.15.Ha

\section{Introduction}

It is usually said that two-dimensional $(1+1)$ gauge theories are trivial. In the continuum, there are no transverse degrees of freedom to sustain any dynamics. Likewise, in the lattice formulation, the system factorizes (after gauge fixing) and a partition function reduces to a simple one-plaquette integral [1-3].

This picture neglects boundary conditions. It is well known [4] that, e.g., Quantum Maxwell Dynamics, $\mathrm{QMD}_{2}$, on a circle with a circumference $L$ is not entirely trivial. There remains one degree of freedom which cannot be gauged away. In the Coulomb gauge, this is the famous, constant in space, mode $A_{x}(t) \equiv A(t)$. Its dynamics is given by the simple, one degree of freedom, Hamiltonian

$$
H=-\frac{e^{2}}{2 L} \frac{d^{2}}{d A^{2}}
$$

Remaining gauge freedom allows to bring $A$ to the interval $[0,2 \pi / L)$, and to identify points $A=0$ and $A=2 \pi / L$, hence the field space is also a 
circle with circumference $L_{A}=2 \pi / L^{1}$. The spectrum and periodic wave functions of the above system are simply

$$
\begin{aligned}
E_{n} & =\frac{1}{2} e^{2} n^{2} L, \quad n=0, \pm 1, \pm 2, \ldots, \\
\psi_{n}(A) & =\frac{1}{\sqrt{L}} e^{i n L A},
\end{aligned}
$$

and describe the quantized states of electric flux which wraps around the circle. These are the straightforward quantum strings, with energies proportional to their length and the string tension $\sigma_{n}=\frac{e^{2} n^{2}}{2}$. These solutions exist without any external charges, the Gauss's law being satisfied due to the non-trivial topology of a circle. Therefore, they are again the simplest examples of topological strings.

In this note, we shall show that this spectrum, and the Hamiltonian can be obtained also from the continuum limit of the standard lattice formulation of $\mathrm{QMD}_{2}$. Moreover, we will derive the generalization of Eq. (1) describing the system of topological strings together with external charges. This will also provide a straightforward, not surprising, but hopefully novel, interpretation of the Manton's parameter $\Theta$ (see also [5]).

Generalizations for arbitrary number of charges as well as for the nonAbelian case will be also outlined.

\section{2. $\mathrm{QMD}_{2}$ on a lattice}

Consider an $N_{t} \times N_{x}$ lattice with unitary variables: $U_{l}=e^{i \theta_{l}}$ associated with spatial link $l$ and $U_{l}=e^{i \vartheta_{l}}$ associated with temporal link $l$. The partition function of this, pure gauge, theory reads

$$
\begin{aligned}
Z & =\int d(\text { links }) \prod_{\text {plaquettes }} B(\text { plaquette }), \\
B(\mathrm{p}) & =e^{\beta \cos \phi_{\mathrm{p}}}
\end{aligned}
$$

with plaquette angles, $\phi_{\mathrm{p}}=\theta_{i}+\vartheta_{j}-\theta_{k}-\vartheta_{l}$, where $\{i, j, k, l\}$ are appropriate indices of links belonging to plaquette ' $\mathrm{p}$ '. This integral is known exactly. Using the character expansion for $\mathrm{U}(1)$, changing variables to plaquette angles, gives ${ }^{2}$

$$
Z=\sum_{n=-\infty}^{\infty} I_{n}(\beta)^{N_{t} N_{x}} .
$$

\footnotetext{
${ }^{1}$ Our $L$ differs by a factor of $2 \pi$ from that of [4].

${ }^{2}$ On two-dimensional, periodic lattices there is one linear constraint between all $N_{t} N_{x}$ plaquettes which has to be taken into account.
} 
To recover the continuum limit, one tunes lattice coupling $\beta$ for each value of lattice constant $a$ according to

$$
\beta=\frac{1}{e^{2} a^{2}},
$$

and expresses all lattice distances in physical units, i.e. $T=a N_{t}, L=a N_{x}$. The natural physical unit which emerges is the dimensionful charge $e$.

Using asymptotic form of modified Bessel functions gives, up to a constant factor,

$$
Z \sim \sum_{n}\left(e^{-\frac{n^{2}}{2 \beta}}\right)^{N_{x} N_{t}}=\sum_{n} e^{-\frac{e^{2} n^{2} L}{2} T}
$$

which proves that indeed the partition function of $\mathrm{QMD}_{2}$ is saturated by topological fluxes Eq. (2).

\subsection{Lattice transfer matrix and Feynman kernel}

It is even more instructive to derive the above equivalence from the transfer matrix formulation. To this end, we employ the Coulomb gauge on the lattice. In fact, the Coulomb condition on the 2-dimensional lattice can be satisfied exactly. Consider a row of horizontal (space-like) link angles $\theta_{1}, \theta_{2}, \ldots, \theta_{N_{x}}$. By local gauge rotation $\alpha_{i}$, they transform to

$$
{ }^{g} \theta_{i} \rightarrow \theta_{i}+\beta_{i}, \quad \beta_{i}=\alpha_{i}-\alpha_{i+1}, \quad \Sigma_{i} \beta_{i}=0 .
$$

Choosing

$$
\beta_{i}=\frac{1}{N_{x}} \sum_{j=1}^{N_{x}} \theta_{j}-\theta_{i},
$$

brings all angles to the same value $\theta=\Sigma_{j} \theta_{j} / N_{x}$, thereby satisfying the Coulomb condition. This procedure fixes all links in one row to the same value, however that value is not fixed since the conditions Eq. (8) leave one rotation angle $\alpha_{i}$ free.

The transfer matrix in this gauge, in the angular representation, is given by the $N_{x}$-fold integral over vertical (time-like) links

$$
\left\langle\theta|\mathcal{T}| \theta^{\prime}\right\rangle=\int \prod_{j=1}^{N_{x}} d \vartheta_{j} \prod_{\mathrm{p}} B(\mathrm{p}),
$$

of the product of $N_{x}$ Boltzmann factors corresponding to all $N_{x}$ plaquettes between two nearest neighbor rows of horizontal links. Due to our gauge 
choice, all plaquettes depend on the same angles $\theta$ and $\theta^{\prime}$, similarly, the states depend only on one angle. Notice also, that since we are not using the temporal gauge, the integrations over the vertical links have to be explicitly included.

Now, we use again the character expansion for Boltzmann factor and integrate over vertical links to obtain

$$
\left\langle\theta|\mathcal{T}| \theta^{\prime}\right\rangle=\sum_{n} I_{n}(\beta)^{N_{x}} e^{i n N_{x}\left(\theta-\theta^{\prime}\right)}
$$

which in the continuum limit becomes

$$
\left\langle\theta|\mathcal{T}| \theta^{\prime}\right\rangle=\sum_{n} e^{-\frac{e^{2}}{2} n^{2} L a} e^{i n L\left(A-A^{\prime}\right)}=K\left(A, A^{\prime}, \epsilon\right) .
$$

This is nothing but a propagator of a 1 DOF quantum mechanical system Eq. (1), (2) over a time $\epsilon=a$.

\section{Topological fluxes with external charges}

\subsection{Eigenenergies}

To place on a circle two external, static charges separated by a distance $R$, consider the correlation function of two Polyakov loops, $\left\langle P(0)^{\dagger} P\left(n_{x}\right)\right\rangle$, separated by $n_{x}$ lattice units. Standard, lattice textbook calculation gives then

$$
Z\left\langle P(0)^{\dagger} P\left(n_{x}\right)\right\rangle=\sum_{n} I_{n}(\beta)^{N_{t}\left(N_{x}-n_{x}\right)} I_{n+1}(\beta)^{N_{t} n_{x}},
$$

which in the continuum limit reads, $R=a n_{x}$,

$$
Z\left\langle P(0)^{\dagger} P\left(n_{x}\right)\right\rangle=\sum_{n} e^{-E_{n}^{P P} T}
$$

with

$$
E_{n}^{P P}=\frac{e^{2}}{2}\left(n^{2}(L-R)+(n+1)^{2} R\right)=\frac{e^{2}(n+\rho)^{2}}{2} L+\frac{e^{2}}{2} L \rho(1-\rho)
$$

and

$$
\rho=\frac{R}{L} .
$$

This result has a simple and appealing interpretation. Time-like Polyakov loops modify Gauss's law at spatial points $x=0$, and $x=R$ - they introduce unit charges at these positions. Such charges cause additional unit of flux extending over a distance $R$. Hence the two contributions to the eigenenergies: an "old" flux over a distance $L-R$ and the "new" one, bigger by one unit (fluxes are additive), over a distance $R$. 
Interesting special cases:

1. at large $T$, lowest state $n=0$ dominates and we have just a standard string of length $R$,

2. with $R=0$, the $n$th energy level is given by topological flux of charge $n$,

3. with $R=L$, i.e. when two charges meet at the "end points" of a circle, they annihilate $\left.\left(e^{+} \delta_{\text {periodic }}(x)+e^{-} \delta_{\text {periodic }}(x-L)\right)=0\right)$ and leave behind a topological string with length $L$ and charge bigger by one unit. In other words, the energy levels are shifted $n \rightarrow n+1$.

Hence, varying the distance $R$ allows us to interpolate between integer valued topological fluxes. This is the meaning of the second representation in Eq. (14). However, the first term is not the whole story (apart from $\rho=0,1)$. There is also the second, constant in $n$, but $R$ and $L$ dependent, contribution which guarantees the linear dependence of eigenenergies on distances involved.

\subsection{The Hamiltonian}

The transfer matrix corresponding to Eq. (12) is similar to Eq. (9)

$$
\left\langle\theta\left|\mathcal{T}_{P P}\right| \theta^{\prime}\right\rangle=\int \prod_{j=1}^{N_{x}} d \vartheta_{j} \prod_{\mathrm{p}} B(\mathrm{p}) e^{-i \vartheta_{1}} e^{i \vartheta_{n_{x}+1}},
$$

except for two additional link variables coming from Polyakov lines $P(1)$ and $P\left(n_{x}+1\right)$. Again, we have chosen the Coulomb gauge, hence the matrix element depends only on two angles $\theta\left(\theta^{\prime}\right)$ which specify a state of an upper (lower) row. As before, upon expanding in characters and integrating over vertical links, one obtains

$$
\left\langle\theta\left|\mathcal{T}_{P P}\right| \theta^{\prime}\right\rangle=\sum_{n} I_{n}(\beta)^{N_{x}-n_{x}} I_{n+1}(\beta)^{n_{x}} e^{i n N_{x}\left(\theta-\theta^{\prime}\right)} e^{i n_{x}\left(\theta-\theta^{\prime}\right)}
$$

which in the continuum limit reads

$$
\begin{aligned}
\left\langle\theta\left|\mathcal{T}_{P P}\right| \theta^{\prime}\right\rangle & =\sum_{n} e^{-\frac{e^{2} L a}{2}\left((n+i \rho)^{2}+\rho(1-\rho)\right)} e^{i(n+\rho) L\left(A-A^{\prime}\right)}=\tilde{K}_{P P}\left(A, A^{\prime}, a\right), \\
\rho & =\frac{R}{L} .
\end{aligned}
$$

This is again a simple Feynman kernel propagating a one DOF system by a time lapse $a$. 
An explicit form of the corresponding Hamiltonian depends on the basis of eigenfunctions we choose. One possibility is

$$
\begin{aligned}
\tilde{H}_{P P} & =\frac{e^{2} L}{2}\left(-\frac{d^{2}}{d \chi^{2}}+\rho(1-\rho)\right), \\
\psi_{n}(\chi) & =e^{i(n+\rho) \chi}, \quad \chi=L A .
\end{aligned}
$$

In this case, wave functions are not periodic. The other choice is

$$
\begin{aligned}
H_{P P} & =\frac{e^{2} L}{2}\left(-\left(\frac{d}{d \chi}+i \rho\right)^{2}+\rho(1-\rho)\right), \\
\psi_{n}(\chi) & =e^{i n \chi}
\end{aligned}
$$

with periodic eigenfunctions. The two are related by the transformation $\psi \rightarrow e^{i \rho \chi} \psi$, which, in general, is not periodic.

This freedom corresponds exactly to the ambiguity discussed in [4] and [6], with Manton's parameter $\Theta$ acquiring now a straightforward interpretation

$$
\Theta=\frac{R}{L}
$$

Namely, as always said, $\frac{e^{2}}{2} \Theta$ represents the external electric field in this context. In the original theory, in the finite volume $L$, the field extends over a fraction $R / L$ of a whole volume. However, in our 1 DOF system the notion of the spatial distance is lost. Therefore, (20) represents an effective field in the reduced model, i.e. the field in the extended $\mathrm{QMD}_{2}$, but averaged over the whole volume $L$. In fact, the equivalence discussed above is the simplest example of the dimensional reduction so successful in many studies [7-14].

\section{Generalizations and summary}

Two extensions immediately suggest themselves.

One, is to add many different, static charges (with total charge being zero) in various positions. Corresponding lattice correlation functions of many time-like Polyakov loops can be readily calculated analogously to Eq. (12). For example, four charges with different magnitudes will be described by

$$
\begin{aligned}
& Z\left\langle P(i)^{\dagger} P(j)^{2 \dagger} P^{2}\left(j+n_{2}\right) P\left(i+n_{1}\right)\right\rangle \\
& =\sum_{n} I_{n}(\beta)^{N_{t}\left(N_{x}-n_{1}-n_{2}\right)} I_{n+1}(\beta)^{N_{t}\left(n_{1}-n_{2}\right)} I_{n+3}(\beta)^{N_{t} n_{2}},
\end{aligned}
$$


with doubly charged sources located inside the single charged ones, i.e. $R_{2} \leq$ $R_{1} \leq L$. Repeating above calculations leads to the following eigenenergies in the continuum limit

$$
E_{n}^{P P}=\frac{e^{2}}{2}\left(n^{2}\left(L-R_{1}-R_{2}\right)+(n+1)^{2}\left(R_{1}-R_{2}\right)+(n+3)^{2} R_{2}\right)
$$

etc. Corresponding 1 DOF quantum mechanical systems can be also readily constructed. This time $\Theta=\left(R_{1}+2 R_{2}\right) / L$, i.e. it is again equal to the external field averaged over the whole volume.

Second generalization is for the non-Abelian pure gauge theory, $\mathrm{QYMD}_{2}$, with arbitrary number of colors. The lattice solutions Eq. (4) and Eq. (12) are basically the same with Bessel functions replaced by the coefficients of the character expansions of Boltzmann factors for an $\mathrm{SU}(N)$ gauge group. Corresponding continuum energies follow from the large $\beta$ behavior of these coefficients.

To summarize, the spectrum of topological fluxes, predicted by Manton quite some time ago, can be also obtained from the continuum limit of seemingly trivial two-dimensional, Abelian lattice gauge theory. The Hamiltonian of the corresponding reduced system also follows from the lattice formulation in Coulomb gauge. Addition of external charges on a lattice leads again to a simple 1 DOF quantum system. Resulting Hamiltonian is, up to a new constant term, the same as Manton's one with non-zero $\Theta$ parameter, which acquires a straightforward interpretation $\Theta=R / L$. To our knowledge, such formula has never been derived before. Generalizations for many charges and for non-Abelian theories were also briefly outlined. We plan to discuss these issues in more detail elsewhere.

This work was partially supported through the National Science Centre grant No. 2011/03/D/ST2/01932, by the Foundation for Polish Science MPD Programme co-financed by the European Regional Development Fund, agreement No. MPD/2009/6, and by the Faculty of Physics, Astronomy and Applied Computer Science, Jagiellonian University Grant No. DSC $/ 000700 / 2012$.

\section{REFERENCES}

[1] T. Banks, J. Kogut, L. Susskind, Phys. Rev. D13, 1043 (1976).

[2] H.J. Rothe, Lattice Gauge Theories: an Introduction, World Scientific, Lecture Notes in Physics, vol. 74 (2005).

[3] D.J. Gross, E. Witten, Phys. Rev. D21, 446 (1980).

[4] N.S. Manton, Ann. Phys. 159, 220 (1985). 
[5] S. Coleman, R. Jackiw, L. Susskind, Ann. Phys. 93, 267 (1975).

[6] M. Asorey, J.G. Esteve, A.F. Pacheco, Phys. Rev. D27, 1852 (1983).

[7] J.D. Bjorken, Elements of Quantum Chromodynamics, SLAC-PUB-2372 (1979).

[8] L. Brink, J. Schwarz, J. Scherk, Nucl. Phys. B121, 77 (1977).

[9] M. Claudson, M.B. Halpern, Nucl. Phys. B250, 689 (1985).

[10] M. Lüscher, Nucl. Phys. B219, 233 (1983).

[11] T. Banks, W. Fischler, S. Shenker, L. Susskind, Phys. Rev. D55, 6189 (1997).

[12] J. Wosiek, Nucl. Phys. B644, 85 (2002).

[13] P. Korcyl, J. Math. Phys. 52, 042102 (2011).

[14] M. Trzetrzelewski, PoS LAT2005, 275 (2006). 